\title{
Genetic parameters for direct and maternal effects on growth traits of sheep
}

\author{
Farhad Ghafouri-Kesbi and Hasan Baneh \\ Islamic Azad University, Karaj Branch, Karaj, Iran
}

\begin{abstract}
The aim of the present study was to estimate (co)variance components and corresponding genetic parameters for birth weight (BW), weaning weight (WW), 6-month weight (W6), 9-month weight (W9), average daily gain from birth to weaning (WWDG), average daily gain from weaning to 6 months (W6DG) and average daily gain from 6 months to 9 months (W9DG) for a nucleus flock of Iranian Makooei sheep. Genetic parameters were estimated by REML procedure fitting six animal models including various combinations of maternal effects. The Akaike information criterion (AIC) was used to determine the most appropriate model. Estimates of direct heritability $\left(h^{2}\right)$ ranged from 0.13 (W6DG) to 0.32 (BW). Maternal effects were found to be important in the growth performance of the Makooei sheep, indicating the necessity of including maternal effects in the model to obtain accurate estimates of direct heritability. Estimates of maternal heritability $\left(\mathrm{m}^{2}\right)$ ranged from 0.05 (W6) to 0.16 (WWDG) and the estimates of proportion of maternal permanent environmental variance to phenotypic variance $\left(c^{2}\right)$ were in the range between $0.05(\mathrm{BW})$ and $0.10(\mathrm{~W} 6)$. Direct additive genetic correlations were positive in all cases and ranged from 0.00 (BW/W9DG) to 0.99 (WW/WWDG). Phenotypic correlations showed a broad range from -0.27 (WW/W9DG) to 0.99 (WW/WWDG). Estimates of genetic parameters showed that genetic improvement through selection programs is possible. WW would be a suitable selection criterion since it has acceptable direct heritability and relatively high genetic correlation with other traits.
\end{abstract}

Keywords: sheep, animal model, REML, heritability, body weight

\section{Introduction}

Contrary to western countries in which consumers show a great preference for beef and pork, in Middle East countries, mutton has always been preferred to other sources of red meat. Small ruminants, i.e., sheep and goat, are numerically important domesticated animals in Iran and traditionally risen on smallholder farms, utilising the range as the major source of feed. In Iran, the primary aim of the breeding programs in sheep production is increasing the efficiency of meat production because of an increasing demand for animal protein as a direct consequence of population growth and improvement in the level of living.

The improving growth performance through selection programs is an important way to increase meat output in lamb production systems. However, selection for growth is complicated by the fact that in mammal species traits related to growth are determined not only by the animal's own additive genetic merit but also by maternal effects. Ghafouri-Kesbi 
\& Eskandarinasab (2008) in sheep, Barazandeh et al. (2011) in goat and Utrera (2008) in cattle demonstrated that animal models which ignore maternal effects might result in overestimation of direct heritability. A direct consequence of such parameter overestimation will be a similar upward bias in predicted responses to selection measured. Using appropriate models including both direct and maternal effects can result in a more accurate estimation of variance components and provide more accurate predictive ability of selection response than would a model that contains only direct genetic effects (Ghafouri-Kesbi \& Eskandarinasab 2008).

The Makooei, numbering about three million heads, is one of the major indigenous sheep breeds of Iran. The animals of this breed are known with medium size, fat tail, carpet wool and white colour. This breed has its origins in the western provinces of Iran which are known as Eastern and Western provinces Azerbaijan. Also, they are reared in Turkey and called White Karaman (Tavakkolian 1999). In the early 1990s, a nucleus flock of Makooei sheep was established at Makoo, Western Azerbaijan, Iran. The aim was to establish a nucleus source for improving other flocks in the region. From the time when first lambs were born, information on growth performance has been recorded and stored in the station. In spite of the presence of this good database, information on genetic parameters for growth traits of Makooei sheep is exceedingly limited. Albeit plenty of genetic information for growth traits has been published in the literature (see for example Safari et al. [2005] and references therein), they are not expandable to Makooei sheep because estimation of genetic parameters is affected by several factors such as breed differences, size and structure of data, etc. The present study, therefore, was conducted to estimate (co)variance components due to direct and maternal effects for traits related to growth of Makooei sheep. Results will guide the researchers to carry out correct breeding strategies for further development of the trait of interest.

\section{Material and methods}

\section{Data}

Data available for analysis were collected on Makooei lambs at the Makooei Sheep Breeding Station at Makoo, Western Azerbaijan, Iran $\left(36^{\circ}, 35^{\prime} \mathrm{S}\right.$ and $\left.48^{\circ}, 22^{\prime} \mathrm{E}\right)$. Climatically, this location has temperate summers and cold winters and receives a mean annual rainfall of about $400 \mathrm{~mm}$. Ewes are raised in an annual breeding cycle starting in September. Young ewes are first mated so as to lamb for the first time at approximately 1.5 years of age. In the mating season, initially artificial insemination (Al) is performed, but animals that do not conceive by $\mathrm{Al}$ are allocated to natural service. In the latter case, ewes are assigned to ram breeding groups with an average mating ratio of 10-15 ewes per ram. Lambing take places from midJanuary to April. At birth, the relevant information about newborn such as sex, birth type, birth date, birth weight, sir ID and dam ID are recorded. Ewes are supplemented, depending upon ewes' requirements for a few days after lambing. During the suckling period, lambs are fed with ewes' milk and also allowed to access to dry alfalfa after 3 weeks of age. Lambs are weaned on average age of 100 days. After weaning, ewes and young animals are kept on natural pasture as separate flocks. They are kept indoors during winter and received a ration composed of wheat straw, dry alfalfa, minerals and some available concentrates. Ewes are kept in the flock for a maximum of 7 parities and rams for 2 or 3 breeding seasons. 


\section{Evaluated traits}

The traits studied were: birth weight (BW), weaning weight (WW), 6-month weight (W6), 9-month weight (W9), average daily gain from birth to weaning (WWDG), average daily gain from weaning to 6 months (W6DG) and average daily gain from 6-month to 9 months (W9DG). Weaning weight, 6-month weight and 9-month weight were adjusted to 100, 180 and 270 days of age, using a linear regression calculated for that cohort. The pre- and postweaning daily gain were calculated as total gain divided by the number of days in the period.

\section{Statistical analysis}

Initially, fixed linear models were applied to the data using Proc GLM in SAS 9 (SAS Institute Inc., Cary, NC, USA) for identifying non-genetic factors to be included in the models. Fixed model included effects for year and month of lambing, sex, birth type and age of dam at lambing. All fixed effects were significant $(P<5 \%)$ for BW, WW, W6, W9 and WWDG, while final fixed models for W6DG and W9DG included fixed effects of year of birth, month of birth and sex of lambs.

Six uni-variate animal models were fitted for each trait to estimate (co)variance components and corresponding genetic parameters. By ignoring or including maternal genetic effect and maternal permanent environmental effect, the following six different models were used: Model I was a model, with direct additive genetic effect as the only random effect.

$$
\text { Model I } \quad y=X b+Z_{1} a+e
$$

Model II comprised maternal permanent environment effect as an additional random effect.

$$
\text { Model II } \quad y=X b+Z_{1} a+Z_{2} c+e
$$

Models III and IV included an additive maternal effect fitted as second random effect but allowing a genetic covariance between direct and maternal genetic effects ( $\operatorname{Cov}(\mathbf{a}, \mathbf{m})$ only for Model IV).

$$
\begin{array}{lll}
\text { Model III } & y=X b+Z_{1} a+Z_{3} m+e & \operatorname{Cov}(a, m)=0 \\
\text { Model IV } & y=X b+Z_{1} a+Z_{3} m+e & \operatorname{Cov}(a, m)=A \sigma_{a, m}
\end{array}
$$

Models $\mathrm{V}$ and $\mathrm{VI}$ included maternal permanent environmental and maternal genetic effects, ignoring and fitting, respectively, direct-maternal genetic covariance.

$$
\begin{array}{lll}
\text { Model V } & y=X b+Z_{1} a+Z_{2} c+Z_{3} m+e & \operatorname{Cov}(a, m)=0 \\
\text { Model VI } & y=X b+Z_{1} a+Z_{2} c+Z_{3} m+e & \operatorname{Cov}(a, m)=A \sigma_{a, m}
\end{array}
$$

where $y$ is the vector of observations, $\boldsymbol{b}$ is the vector of fixed effects, $\boldsymbol{a}, \boldsymbol{m}, \boldsymbol{c}$ and $\boldsymbol{e}$ are the vectors of direct additive genetic effect, maternal genetic effect, maternal permanent environmental effect and the residual (temporary environment) effect, respectively. $X, Z_{\gamma^{\prime}}$ $Z_{2}$ and $Z_{3}$ are incidence matrices relating individual records to $b, a, c$ and $m$, respectively. The covariance structure for the model was:

$$
V(a)=A \sigma_{a^{\prime}}^{2}, V(m)=A \sigma_{m^{\prime}}^{2}, V(c)=I_{n} \sigma_{c^{\prime}}^{2} V(e)=I_{e} \sigma_{e}^{2} \text { and } \operatorname{Cov}(a, m)=A \sigma_{a, m}
$$

where $I_{n}$ and $I_{e}$ are identity matrices of order equal to the number of dams and number of records, respectively. $\sigma_{a^{\prime}}^{2} \sigma_{m}^{2}, \sigma_{c}^{2}$ and $\sigma_{e}^{2}$ are direct additive genetic variance, maternal genetic 
variance, maternal permanent environmental variance, and residual variance, respectively, and $\sigma_{a, m}$ is direct-maternal genetic covariance.

DFREML program of Meyer (2000) was used to estimate genetic parameters with a REML algorithm. A variance of $10^{-8}$ of Simplex function values was chosen as the convergence criterion which gives a good accuracy of estimation.

Traditionally log-likelihood ratio tests (LRT) are used to determine the most appropriate model by comparing the differences between log-likelihoods (-2logL) to a critical value from a chi-square distribution. Using LRT, only models that differ by at least one parameter are comparable, i.e., comparison of model 2 with model 3 is not feasible by LRT because both models include the same number of parameters. For this reason, the Akaike information criterion (AIC) of Akaike (1973) was computed to rank the models. Let p denote the number of random (co)variance parameters to be estimated and $L$ og $L$ is the maximum likelihood, then the information criterion is defined as: $\mathrm{AIC}=-2 \mathrm{Log} L+2 \mathrm{p}$. The model yielding the smallest AIC fits the data best.

The bi-variate animal models which included the same fixed effects as uni-variate models were used to estimate covariances between each pair of traits. The models applied in twotrait analyses were those fitted for each of the underlying traits in the single-trait analyses.

\section{Results and discussion}

Characteristics of the data structure are summarised in Table 1. Different growth rates were observed in the various growth phases in a way that average daily gain during pre-weaning period was 2 times as great as that from weaning to 6 months of age and 3 times as great as that observed between 6-month and 9-month. Similarly, Prince et al. (2010) in Avikalin sheep and Ghafouri-Kesbi et al. (2011) in Zandi sheep observed maximum growth rate in the preweaning period. The difference between pre- and post-weaning growth rate might attribute to change in nutritional conditions after weaning caused by terminating of suckling. Among traits studied, BW had the minimum CV. Miraei-Ashtiani et al. (2007) in Sangsari sheep and Eskandarinasab et al. (2010) in Afshari sheep reported similar findings. The reason of less CV for BW may be due to less variation and effect of outside environment on this trait, as ewes were kept inside and manually fed in the late months of pregnancy period in the winter (MiraeiAshtiani et al. 2007). According to Salako (2006), larger variation within certain measurements suggests absence of selection, or the parts respond more to environment than others.

AIC values for the different models are presented in Table 2. Except for W9DG, on which maternal effects had no significant effect, for other traits studied, considering maternal effects fitted the data substantially better than the simple additive model (Model I). For BW, the considerable increase in likelihood appeared in Model II with the maternal permanent environmental effects fitted as the only additional random effect. For WW and W6, the likelihood values increased even further by partitioning the phenotypic variance into direct additive genetic, maternal genetic, and maternal permanent environmental components (Model V). For W9, by including maternal permanent environmental component the likelihood increased significantly and so Model II was determined as the most appropriate model. The most appropriate model for WWDG and W6DG which described the data best included maternal genetic effect and maternal permanent environmental effect, respectively (Models III and II, respectively). For W9DG, the model including only direct additive effect 
(Model I) was sufficient to explain the variation in the data as addition of maternal effects did not improve the likelihood than Model I. Improved fit of analytical models by the inclusion of maternal effects has been reported by other researchers (Abegaz et al. 2005, Ghafouri-Kesbi \& Eskandarinasab 2008, Kariuki et al. 2010, Ghafouri-Kesbi et al. 2011). It is notable that data structure has a great impact on the accuracy of maternal effects estimation. With a small data set and shallow pedigree, maternal effects cannot be separated from direct effects very well. For accurately separating maternal genetic components and maternal permanent environmental effects from combined and direct effects, a large dataset and several welllinked generations of records and many relationship between relatives related to the mother are needed. Only after meeting these requirements, accurate estimates of maternal effects would be obtained (Maniatis \& Pollott 2003).

Table 1

Characteristics of the data structure

\begin{tabular}{|c|c|c|c|c|c|c|c|}
\hline & $\mathrm{BW}, \mathrm{kg}$ & WW, kg & W6, kg & W9, kg & WWDG, $\mathrm{g}$ & W6DG, g & W9DG, $g$ \\
\hline No. of records & 1115 & 1062 & 1472 & 1151 & 1062 & 674 & 570 \\
\hline No. of sires with progeny & 68 & 71 & 72 & 71 & 71 & 76 & 61 \\
\hline No. of dams with progeny & 444 & 445 & 599 & 514 & 445 & 356 & 315 \\
\hline No. of grandsires with progeny & 76 & 76 & 66 & 42 & 76 & 59 & 57 \\
\hline No. of granddams with progeny & 271 & 268 & 249 & 210 & 268 & 219 & 210 \\
\hline No. of sires with own record & 14 & 14 & 19 & 17 & 14 & 11 & 10 \\
\hline No. of dams with own record & 158 & 155 & 275 & 248 & 155 & 86 & 75 \\
\hline Average no. of progeny per sire & 12.35 & 11.99 & 19.01 & 15.31 & 11.52 & 8.89 & 7.87 \\
\hline Average no. of progeny per dam & 2.49 & 2.41 & 2.58 & 2.30 & 2.39 & 1.91 & 1.71 \\
\hline Mean & 4.251 & 12.283 & 25.377 & 27.517 & 170.146 & 73.437 & 49.394 \\
\hline SD & 0.587 & 3.890 & 4.263 & 4.760 & 36.284 & 48.180 & 28.126 \\
\hline $\mathrm{CV}, \%$ & 13.81 & 18.27 & 16.79 & 17.29 & 12.32 & 65.60 & 56.94 \\
\hline
\end{tabular}

BW: birth weight, WW: weaning weight (100 days), W6: 6 months weight (180 days), W9: 9 months weight (270 days), WWDG: average daily gain from birth to weaning, W6DG: average daily gain from weaning to 6 months, W9DG: average daily gain from 6 months to 9 months of age, SD: standard deviation, CV: phenotypic coefficient of variation

Table 2

AIC values and direct heritability estimates from six uni-variate analyses for each trait (best model: bold)

\begin{tabular}{lcccccccccccccc}
\hline & \multicolumn{3}{c}{ BW } & \multicolumn{3}{c}{ WW } & \multicolumn{2}{c}{ W6 } & \multicolumn{2}{c}{ W9 } & \multicolumn{3}{c}{ WWDG } & \multicolumn{2}{c}{ W6DG } & \multicolumn{2}{c}{ W9DG } \\
Model & AIC & $h^{2}$ & AIC & $h^{2}$ & AIC & $h^{2}$ & AIC & $h^{2}$ & AIC & $h^{2}$ & AIC & $h^{2}$ & AIC & $h^{2}$ \\
\hline I & -684.66 & 0.38 & 3270.70 & 0.42 & 4815.46 & 0.38 & 3678.22 & 0.37 & 7297.84 & 0.40 & 5221.14 & 0.18 & $\mathbf{3 6 2 6 . 7 8}$ & $\mathbf{0 . 1 8}$ \\
II & $-\mathbf{6 7 5 . 1 2}$ & $\mathbf{0 . 3 2}$ & 3252.30 & 0.24 & 4793.18 & 0.26 & $\mathbf{3 6 7 6 . 2 4}$ & $\mathbf{0 . 2 8}$ & 7910.28 & 0.20 & $\mathbf{5 2 2 0 . 1 0}$ & $\mathbf{0 . 1 3}$ & 3627.96 & 0.18 \\
III & -682.66 & 0.31 & 3248.94 & 0.22 & 4792.34 & 0.21 & 3677.22 & 0.29 & $\mathbf{7 9 0 9 . 2 5}$ & $\mathbf{0 . 1 7}$ & 5221.18 & 0.13 & 3628.56 & 0.19 \\
IV & -680.75 & 0.29 & 3250.36 & 0.28 & 4794.30 & 0.21 & 3678.44 & 0.32 & 7910.74 & 0.22 & 5220.30 & 0.20 & 3628.44 & 0.21 \\
V & -681.22 & 0.31 & $\mathbf{3 2 4 8 . 7 6}$ & $\mathbf{0 . 2 1}$ & $\mathbf{4 7 8 7 . 8 4}$ & $\mathbf{0 . 2 2}$ & 3678.98 & 0.28 & 7909.26 & 0.17 & 5222.08 & 0.12 & 3629.09 & 0.19 \\
VI & -679.60 & 0.37 & 3250.12 & 0.27 & 4789.78 & 0.24 & 3678.20 & 0.31 & 7910.68 & 0.22 & 5221.00 & 0.20 & 3629.14 & 0.21 \\
\hline
\end{tabular}

BW: birth weight, WW: weaning weight (100 days), W6: 6 months weight (180 days), W9: 9 months weight (270 days), WWDG: average daily gain from birth to weaning, W6DG: average daily gain from weaning to 6 months, W9DG: average daily gain from 6 months to 9 months of age

Results of the uni-variate analyses based on the most appropriate models are shown in Table 3. The estimates of direct heritability $\left(h^{2}\right)$ for traits studied were in the range between 0.13 (W6DG) and 0.32 (BW). These estimates show the presence of heritable variation in the growth traits of Makooei sheep. In spite of increase in direct additive genetic variance with age (from 0.06 at birth to 2.645 at 9 months of age), estimates of direct heritability for WW, W6 and W9 affected by greater contribution of other maternal and environmental components in 
the phenotypic variance. Because estimation of heritability is affected by several factors such as genetic structure of the population, management conditions and method of estimation, it appears difficult to compare current results with results from the literature. However, current estimates of $h^{2}$ are within the range of reports from other Iranian sheep breeds (MiraeiAshtiani et al. 2007, Bahreini-Behzadi et al. 2007, Ghafouri-Kesbi \& Eskandarinasab 2008, Eskandarinasab et al. 2010, Ghafouri-Kesbi et al. 2011). Also they fall within the range reported by Safari et al. (2005) for several sheep breeds word wide. In general, moderate estimates of heritability for the growth traits indicate scope for genetic improvement in Makooei sheep through selection for growth traits.

Table 3

Estimates of (co)variance components and genetic parameters (SE) from uni-variate analyses based on the most appropriate models

\begin{tabular}{lcccccrcccc}
\hline & Model & $\sigma_{a}^{2}$ & $\sigma_{m}^{2}$ & \multicolumn{1}{c}{$\sigma_{c}^{2}$} & $\sigma_{e}^{2}$ & \multicolumn{1}{c}{$\sigma_{p}^{2}$} & \multicolumn{1}{c}{$h^{2}$} & $m^{2}$ & $c^{2}$ & $h_{t}^{2}$ \\
\hline BW & II & 0.060 & - & 0.009 & 0.115 & 0.185 & $0.32(0.08)$ & - & $0.05(0.03)$ & 0.32 \\
WW & V & 1.817 & 0.704 & 0.726 & 5.254 & 8.502 & $0.21(0.08)$ & $0.08(0.06)$ & $0.08(0.06)$ & 0.25 \\
W6 & V & 2.277 & 0.569 & 1.069 & 6.292 & 10.208 & $0.22(0.07)$ & $0.05(0.04)$ & $0.10(0.04)$ & 0.25 \\
W9 & II & 2.645 & - & 0.868 & 5.975 & 9.490 & $0.28(0.09)$ & - & $0.09(0.04)$ & 0.28 \\
WWDG & III & 140.86 & 134.49 & - & 535.476 & 810.839 & $0.17(0.08)$ & $0.16(0.04)$ & - & 0.27 \\
W6DG & II & 134.594 & - & 65.869 & 878.108 & 1076.571 & $0.13(0.08)$ & - & $0.06(0.04)$ & 0.13 \\
W9DG & I & 48.437 & - & - & 220.877 & 269.315 & $0.18(0.09)$ & - & - & 0.18 \\
\hline
\end{tabular}

$\sigma_{a}^{2}$ direct additive genetic variance, $\sigma_{m}^{2}$ :maternal additive genetic variance, $\sigma_{c}^{2}$ : maternal permanent environmental variance, $\sigma_{e^{2}}^{2}$ residual variance, $\sigma_{p}^{2}$ : phenotypic variance, $h^{2}$ : direct heritability, $m^{2}$ : maternal heritability, $c^{2}$ : ratio of maternal permanent environmental variance to phenotypic variance, $h_{t}^{2}$ total heritability (formula [8]) BW: birth weight, WW: weaning weight (100 days), W6: 6 months weight (180 days), W9: 9 months weight (270 days), WWDG: average daily gain from birth to weaning, W6DG: average daily gain from weaning to 6 months, W9DG: average daily gain from 6 months to 9 months of age

Except for W9DG, other traits influenced by maternal effects. Maternal effects in sheep have been extensively studied in recent years (see e.g. Szwaczkowski et al. 2006, GhafouriKesbi \& Eskandarinasab 2008, Prince et al. 2010). These researchers emphasised on inclusion of maternal effects in the model to obtain accurate estimates of direct heritability. The estimates of maternal heritability $\left(\mathrm{m}^{2}\right)$ were in the range between 0.05 (W6) and 0.16 (WWDG) which were close to those reported by Abegaz et al. (2005) in Horro sheep. The importance of maternal genetic effects is further evidenced by estimates of total heritability $\left(h_{t^{\prime}}^{2}\right.$ Willham 1972):

$$
h_{t}^{2}=\frac{\sigma_{a}^{2}+0.5 \sigma_{m}^{2}+1.5 \sigma_{a, m}}{\sigma_{p}^{2}}
$$

Estimates of $h_{t}^{2}$ are of value to predict phenotypic response to selection. According to the $h_{t}^{2}$ formula, when direct-maternal genetic covariance is positive, presence of maternal genetic effects increases the total heritability, and in consequence, the potential response to selection (Ghafouri-Kesbi \& Eskandarinasab 2008). As shown in Table 3, estimates of $h_{t}^{2}$ are higher than estimates of $h^{2}$, indicating that phenotypic response to selection would be higher than that predicted using estimates of $h^{2}$. The estimates of $c^{2}$ (the permanent environmental variance due to dam as a proportion of phenotypic variance) ranged from 0.05 (BW) to 0.10 (W6) which are comparable with those in the literature (Ghafouri-Kesbi \& Eskandarinasab 2008, Prince et al. 2010, Kariuki et al. 2010). Estimates of $c^{2}$ are not as informative as $m^{2}$, however, in order to 
obtain accurate estimates of $m^{2}$, estimation of $c^{2}$ is necessary as exclusion of the maternal permanent environmental effects could cause maternal heritability to be overestimated.

Different correlations among traits studied are presented in Table 4. Many of these results are in accord with the findings of other studies (Abegaz et al. 2005, Miraei-Ashtiani et al. 2007, Eskandarinasab et al. 2010, Gowane et al. 2011). The additive genetic correlation between growth traits, arising chiefly from pleiotropy, was positive in all cases ( 0.00 to 0.99$)$, indicating the fact that selection for increased body weight at certain stage would also result in genetic improvement in the subsequent development of body weight, which will be reflected in the average daily weight gain during the pre- and post-weaning stages of development (Gowane et al. 2011). The correlations between adjacent measurements were larger than ones between non-adjacent measurements. For example, the additive genetic correlation is 0.60 between BW and WW, falling slightly to 0.31 between BW and W9. This result is expected because an autocorrelation would exist among the genetic and environmental effects associated with the measurements (Shaat et al. 2004). A result which has been frequently reported by different researchers (e.g., Abegaz et al. 2005, Miraei-Ashtiani et al. 2007, Eskandarinasab et al. 2010) is the negative phenotypic correlation between pre- and post-weaning daily gain in spite of positive genetic correlation. Compensatory growth of some poorly nursed lambs in postweaning period may be the reason for this phenomenon (Abegaz et al. 2005). The positive genetic and phenotypic correlations between WW and other pre- and post-weaning growth traits, makes this trait to be a suitable selection criterion to improve growth performance of the Makooei sheep. Maternal genetic correlations among WW, WWDG and W6 were highly positive, which indicates that the genes of dams which contribute in milk production could also have some favourable effect on post-weaning growth traits.

Table 4

Correlations between traits yielded from bi-variate analyses

\begin{tabular}{llllll}
\hline Trait 1 & Trait 2 & \multicolumn{1}{c}{$r_{a}$} & $r_{m}$ & $r_{c}$ & $r_{p}$ \\
\hline BW & WW & $0.60(0.16)$ & - & $0.29(0.27)$ & 0.26 \\
BW & W6 & $0.42(0.14)$ & - & $-0.62(0.37)$ & 0.19 \\
BW & W9 & $0.31(0.19)$ & - & $-0.23(0.16)$ & 0.19 \\
BW & WWDG & $0.35(0.15)$ & - & - & 0.12 \\
BW & W6DG & $0.02(0.02)$ & - & $0.34(0.53)$ & 0.02 \\
BW & W9DG & $0.00(0.10)$ & - & - & -0.02 \\
WW & W6 & $0.87(0.09)$ & $0.99(0.18)$ & $0.75(0.26)$ & 0.62 \\
WW & W9 & $0.51(0.12)$ & - & $0.52(0.22)$ & 0.55 \\
WW & WWDG & $0.99(0.01)$ & $0.99(0.01)$ & - & 0.98 \\
WW & W6DG & $0.46(0.03)$ & - & $0.08(0.10)$ & -0.16 \\
WW & W9DG & $0.27(0.11)$ & - & - & -0.27 \\
W6 & W9 & $0.44(016)$ & - & $0.32(0.13)$ & 0.46 \\
W6 & WWDG & $0.88(0.10)$ & $0.91(0.08)$ & - & 0.61 \\
W6 & W6DG & $0.70(0.18)$ & - & $0.55(0.27)$ & 0.58 \\
W6 & W9DG & $0.17(0.19)$ & - & - & 0.04 \\
WWDG & W6DG & $0.45(0.21)$ & - & - & -0.15 \\
WWDG & W9DG & $0.08(0.09)$ & - & - & -0.22 \\
W6DG & W9DG & $0.18(0.06)$ & - & - & 0.12 \\
\hline
\end{tabular}

$r_{a}$ : direct additive genetic correlation, $r_{m}$ : maternal genetic correlation, $r_{c}$ : maternal permanent environmental correlation, $r_{p}$ : phenotypic correlation, BW: birth weight, WW: weaning weight (100 days), W6: 6 months weight (180 days), W9: 9 months weight (270 days), WWDG: average daily gain from birth to weaning, W6DG: average daily gain from weaning to 6 months, W9DG: average daily gain from 6 months to 9 months of age 
In conclusion, the results of the current study advocate the idea that to obtain accurate estimates of genetic parameters for growth traits and to increase the accuracy of genetic evaluation, both direct and maternal effects need to be included in the model of analysis. Results obtained here could be used by breeders to plan appropriate breeding programs for improving growth performance of the Makooei sheep. Body weight at weaning seems would be a suitable selection criterion, as it is recorded early in life and has acceptable direct heritability and relatively high genetic correlation with other growth traits. However, maternal effects on WW must not be ignored where lambs are selected based on their genetic value for WW because maternal effects can mask true genetic potential of lambs.

\section{Acknowledgements}

The Makooei Sheep Breeding Station staff for providing the data used in this study are gratefully acknowledged.

\section{References}

Abegaz S, van Wyk JB, Olivier JJ (2005) Model comparisons and genetic and environmental parameter estimates of growth and the Kleiber ratio in Horro sheep. S Afr J Anim Sci 35, 30-40

Akaike H (1973) Information theory and an extension of the maximum likelihood principle. In: Petrov BN, Csaki F (eds) Proceedings of the 2nd International Symposium on Information Theory Akademiai Kiado, Budapest, Hungary, 267-81

Bahreini-Behzadi MR, Shahroudi FE, Van Vleck LD (2007) Estimates of genetic parameters for growth traits in Kermani sheep. J Anim Breed Genet 124, 296-301

Barazandeh A, Molaei Moghbeli S, Vatankhah M, Mohammadabadi M (2012) Estimating non-genetic and genetic parameters of pre-weaning growth traits in Raini Cashmere goat. Trop Anim Health Prod 44, 864-867

Eskandarinasab M, Ghafouri-Kesbi F, Abbasi MA (2010) Different models for evaluation of growth traits and Kleiber ratio in an experimental flock of Iranian fat-tailed Afshari sheep. J Anim Breed Genet 127, 26-33

Ghafouri-Kesbi F, Eskandarinasab MP (2008) An evaluation of maternal influences on growth traits: the Zandi sheep breed of Iran as an example. J Anim Feed Sci 17, 519-529

Ghafouri-Kesbi F, Abbasi MA, Afraz F, Babaei M, Baneh H, Abdollahi Arpanahi R (2011) Genetic analysis of growth rate and Kleiber ratio in Zandi sheep. Trop Anim Health Prod 43, 1153-1159

Gowane GR, Chopra A, Prince LLL, Mishra AK, Arora AL (2011) Genetic analysis for growth traits of prolific Garole $\times$ Malpura (GM) sheep. Trop Anim Health Prod 43, 299-303

Kariuki CM, Ilatsia ED, Kosgey IS, Kahi AK (2010) Direct and maternal (co)variance components, genetic parameters and annual trends for growth traits of Dorper sheep in semi-arid Kenya. Trop Anim Health Prod 42, 473-481

Maniatis N, Pollott GE (2003) The impact of data structure on genetic (co)variance components of early growth in sheep, estimated using an animal model with maternal effects. J Anim Sci 81, 101-108

Meyer K (2000) Programs to estimate variance components for individual animal models by restricted maximum likelihood (REML). Ver. 3.1. Users notes. Institute of Animal Science, Armidale, Australia

Miraei-Ashtiani SR, Seyedalian SAR, Moradi Shahrbabak M (2007) Variance components and heritabilities for body weight traits in Sangsari sheep, using univariate and multivariate animal models. Small Rumin Res 73, 109-114

Prince LLL, Gowane GR, Chopra A, Arora AL (2010) Estimates of (co)variance components and genetic parameters for growth traits of Avikalin sheep. Trop Anim Health Prod 42, 1093-1101 
Safari E, Fogarty NM, Gilmour AR (2005) A review of genetic parameter estimates for wool, growth, meat and reproduction traits in sheep. Livest Prod Sci 92, 271-289

Salako AE (2006) Application of morphological indices in the assessment of type and function in sheep. Int J Morphol 24, 13-18

Shaat I, Galal S, Mansour H (2004) Genetic trends for lamb weights in flocks of Egyptian Rahmani and Ossimi sheep. Small Rumin Res 51, 23-28

Szwaczkowski T, Wójtowski J, Stanisławska E, Gut A (2006) Estimates of maternal genetic and permanent environmental effects in sheep. Arch Tirez 49, 186-192

Tavakkolian J (1999) The genetic resources of native farm animals of Iran. Animal Science Research Institute of Iran Press, Karaj, Iran, 213-218

Utrera AR (2008) Estimates of genetic parameters for pre-weaning growth traits of cattle. Téc Pecu Méx 46, 37-67

Willham RL (1972) The role of maternal effects in animal breeding: III. Biometrical aspects of maternal effects in animals. J Anim Sci 35, 1288-1293

Received 5 May 2012, accepted 10 February 2012.

Corresponding author:

Farhad Ghafouri-Kesbi

email: farhad_ghy@yahoo.com

Islamic Azad University, Karaj Branch, P. O. Box: 31485-313, Karaj, Iran 